\title{
Correction to: Control and Controllability of PDEs with Hysteresis
}

\section{Chiara Gavioli ${ }^{1}$ Pavel Krejčí ${ }^{2,3}$}

Published online: 19 March 2020

(c) Springer Science+Business Media, LLC, part of Springer Nature 2020

\section{Correction to: Appl Math Optim https://doi.org/10.1007/s00245-020-09663-6}

Unfortunately, the original version of the article has contained an error in Equation 4.17 .

The corrected equation is given below.

$$
y_{t}^{r \gamma_{n}} \rightarrow \hat{y}_{t}^{r}, y^{r \gamma_{n}} \rightarrow \hat{y}^{r}, \frac{1}{\gamma_{n}} \Psi^{\prime}\left(\frac{1}{r} y^{r \gamma_{n}}\right) \rightarrow w^{r} \text { weakly in } L^{2}(Q) \text { as } n \rightarrow \infty \text {. }
$$

The original version of this article was revised.

Publisher's Note Springer Nature remains neutral with regard to jurisdictional claims in published maps and institutional affiliations.

The original article can be found online at https://doi.org/10.1007/s00245-020-09663-6.

$\bowtie$ Chiara Gavioli

chiara.gavioli@unimore.it

Pavel Krejčí

krejci@math.cas.cz; Pavel.Krejci@cvut.cz

1 Dipartimento di Scienze Fisiche, Informatiche e Matematiche, University of Modena and Reggio Emilia, via Campi 213/b, 41125 Modena, Italy

2 Institute of Mathematics, Czech Academy of Sciences, Žitná 25, 11567 Praha 1, Czech Republic

3 Faculty of Civil Engineering, Czech Technical University, Thákurova 7, 16629 Praha 6, Czech Republic 